\title{
Sir David Alan Hopwood
}

\author{
Leonard Katz
}

Received: 11 October 2013 / Accepted: 18 October 2013 / Published online: 7 November 2013

(C) Society for Industrial Microbiology and Biotechnology 2013

This special issue of the Journal of Industrial Microbiology and Biotechnology, entitled "Microbial Genome Mining" is dedicated to Sir David Hopwood, in tribute to his extensive contributions to basic and applied research in the field of natural products, and leadership in the field of streptomycete genetics and molecular biology. After completing his undergraduate and doctoral studies at the University of Cambridge, Sir David stayed on at Cambridge as an Assistant Lecturer for 3 years and then took a lectureship at the University of Glasgow. In 1968, he was appointed John Innes Professor of Genetics at the University of East Anglia, and Head of the Genetics Department at the John Innes Center in Norwich, positions he held for 30 years until his official retirement in 1998. He currently holds the titles of John Innes Emeritus Fellow and Emeritus Professor of Genetics at the University of East Anglia. Sir David has spent his entire career working with streptomycetes and, in particular, Streptomyces coelicolor. As a $\mathrm{PhD}$ student, he was the first to demonstrate that this multi-antibiotic-producing bacterium can undergo genetic exchange via natural mating. He isolated auxotrophic mutants of this organism and, using reciprocal three-factor crosses, mapped their genetic locations. In the 1970s, under his leadership, his laboratory and associates at the John Innes Center began to develop the tools of molecular biology for streptomycetes, which laid the groundwork for the ability of his laboratory and others around the world to genetically manipulate these organisms to produce, or overproduce, native, hybrid, and novel secondary metabolites. This period marked the development of methods for the transformation as well as fusion of protoplasts, and construction

L. Katz $(\bowtie)$

Emeryville, CA, USA

e-mail: katzl@berkeley.edu of numerous plasmid and phage vectors carrying drugresistance markers that proved useful in many actinomycete hosts, including industrially important ones. The laboratory manual produced by the staff of the John Innes Genetics Department in 1985 entitled "Genetic Manipulation of Streptomyces: a Laboratory Manual", followed in 2000 by "Practical Streptomyces Genetics" became and remain the bibles of all laboratories that work with actinomycetes. At the heart of this work was Sir David's interest in the genetic control of biosynthesis of secondary metabolites. His group was the first to show, from mapping studies, that antibiotic biosynthesis genes are clustered, and the first to sequence an antibiotic biosynthesis gene cluster. This work uncovered regulatory genes among the biosynthesis cluster. Global regulatory genes, affecting not only antibiotic biosynthesis but also development, were also found during this period. Complete sequence analysis of an entire cluster established the concept that all the genes required for the biosynthesis of a specific antibiotic can be moved as a single linkage group from one organism to another. This led to work, described in a 1985 landmark Nature paper, performed in collaboration with Professors Heinz Floss and Satoshi Omura, to the generation of the first hybrid antibiotics, produced exclusively through gene cloning of biosynthesis genes from different Streptomyces hosts. In the 1990s, while whole-genome sequencing projects centered on bacterial pathogens or Archaea, which were approximately 4 megabases or smaller, Sir David single-handedly took on the task of obtaining funding to have the $S$. coelicolor genome fully sequenced. His laboratory constructed an ordered set of overlapping cosmids spanning the entire 7.8 million nucleotides of the genome, still among the largest bacterial genomes to have been so mapped, that served as the source from which the sequence was obtained-a project completed at the Sanger Center 
in 2002 that he personally oversaw. Sequence of the full genomes of S. coelicolor and Streptomyces avermitilis, the avermectin producer, generated a few months earlier at the Kitasato Institute using a shotgun sequencing approach, confirmed that secondary metabolite biosynthetic genes are clustered. Equally as important, the sequences revealed that these organisms contain many additional biosynthetic gene clusters encoding natural products in their genomes that are not expressed under standard laboratory conditions of cultivation. The work reported by these two groups established the value of "genome mining" that, together with recent advances in DNA sequencing, DNA synthesis, and analytical chemistry, form the basis for the current rebirth in interest in the discovery of new natural products that is taking place in academic labs, with new funding from government agencies, as well as in emerging companies. The Society for Industrial Microbiology and Biotechnology is honored and privileged, therefore, to dedicate this special issue of JIMB devoted to the new field of genome mining to Sir David Hopwood, for his remarkable research and leadership that has made this possible.

Leonard Katz

President, Society for Industrial Microbiology 